\title{
UDC 663.5
}

SCOPUS CODE 1305

https://doi.org/10.36073/1512-0996-2019-4-27-33

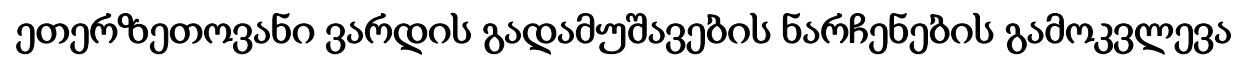

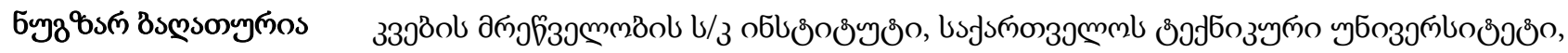

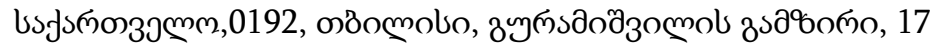

E-mail: nugzi@yahoo.com

mono\%s jszosos

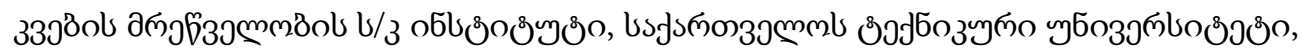

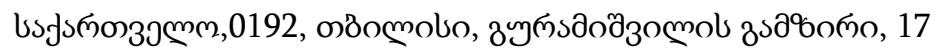

E-mail: I.qajaia@gtu.ge

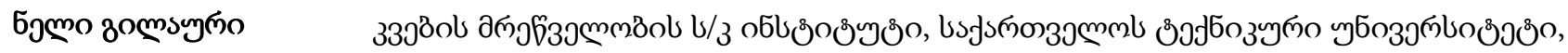

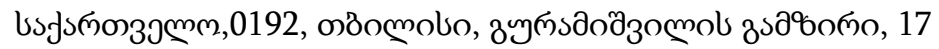

E-mail: n.gilauri@gtu.ge

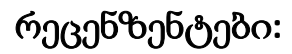

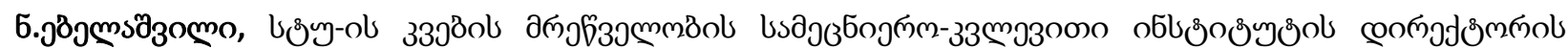

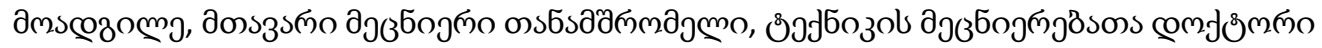

E-mail: nana-ebelashvili@hotmail.com

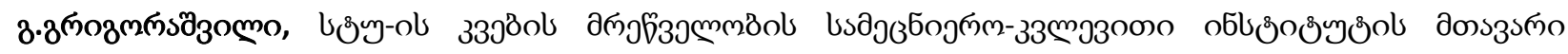

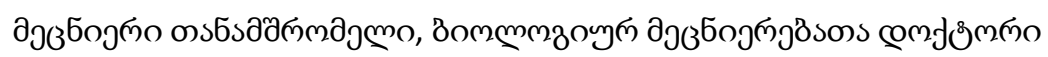

E-mail: g.grigorashvili@gtu.ge

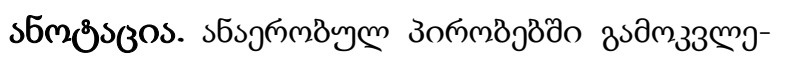

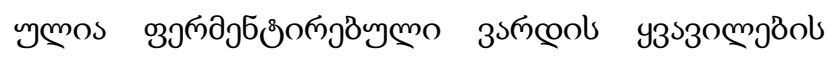

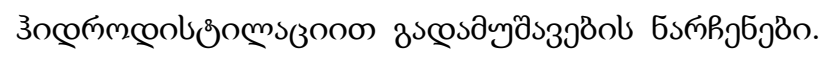

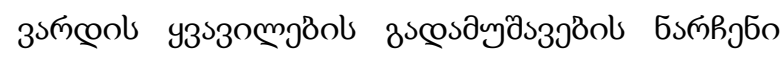

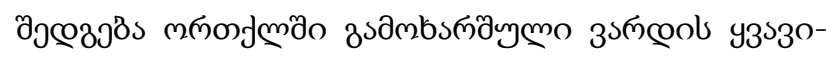

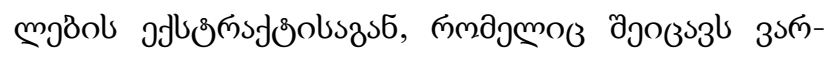

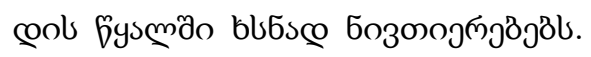

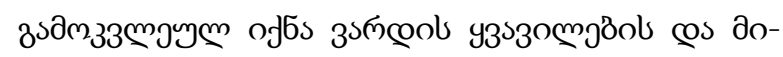

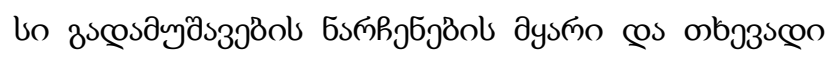

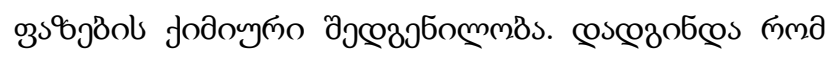

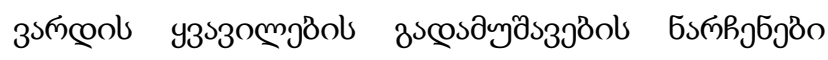

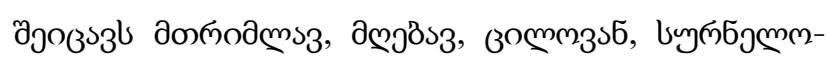

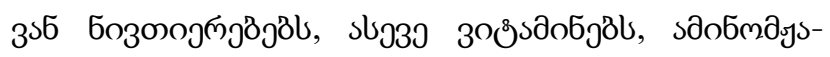

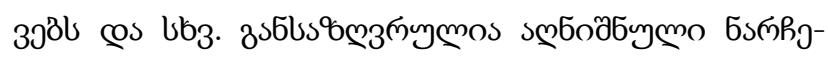

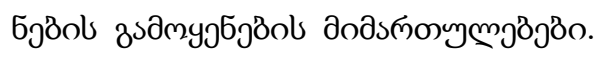

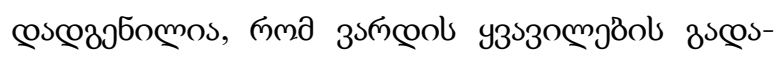

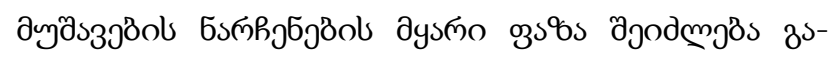

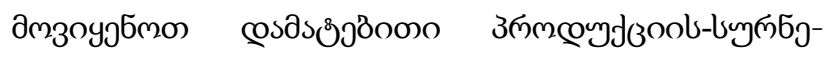

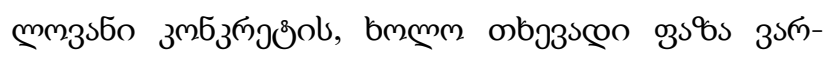

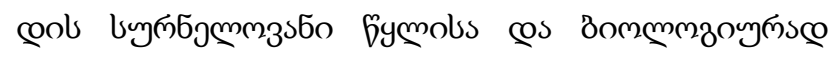

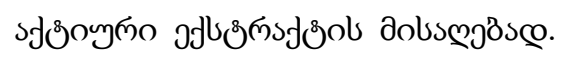




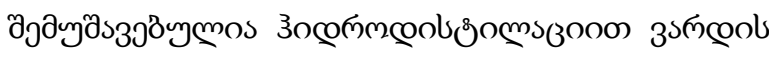

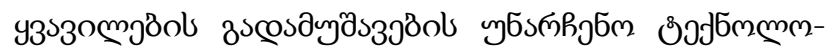

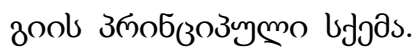

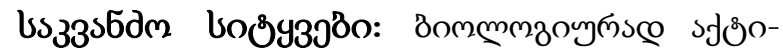

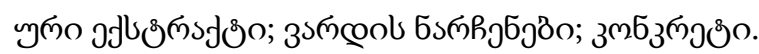

\section{gjuszsmo}

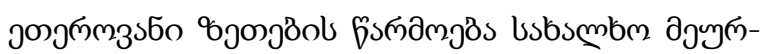

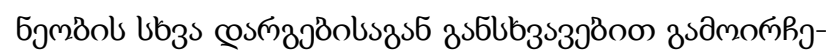

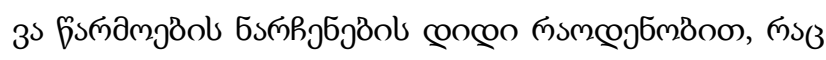

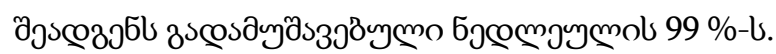

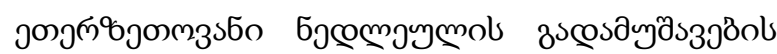

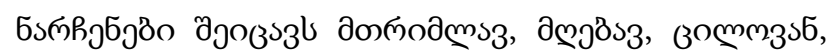

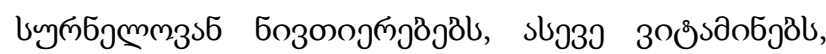

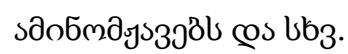

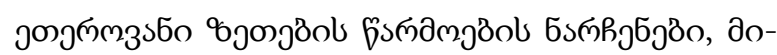

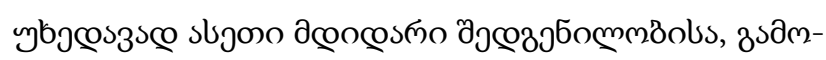

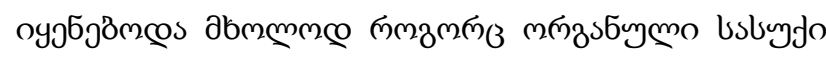

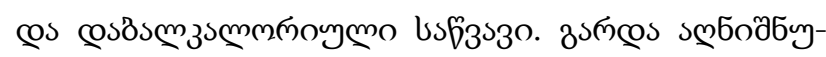

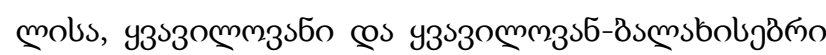

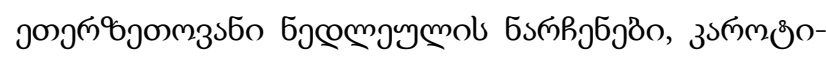

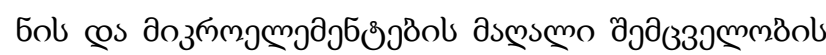

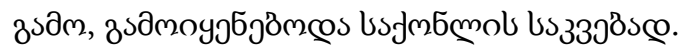

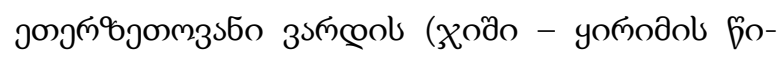

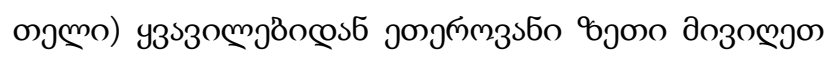

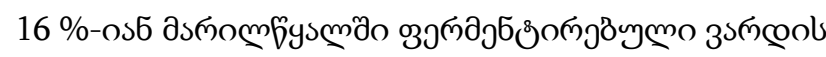

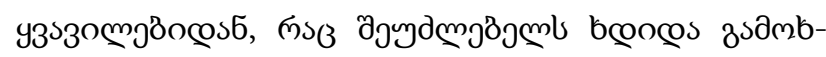

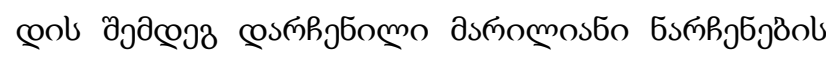

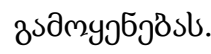

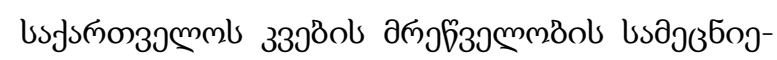

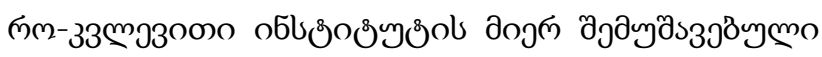

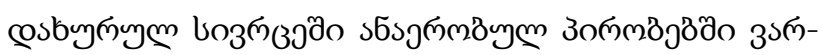

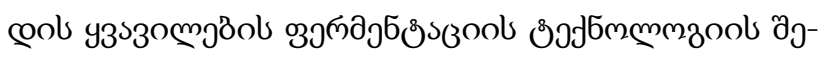

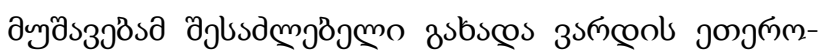

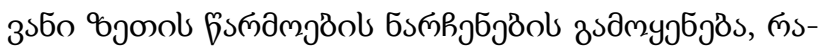

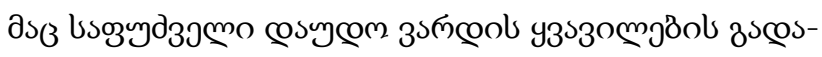

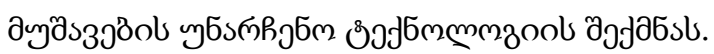

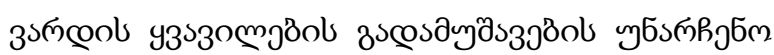

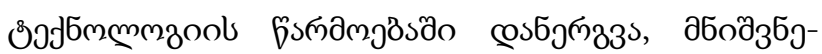

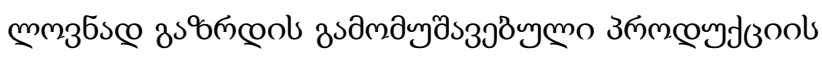

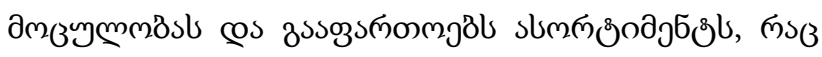

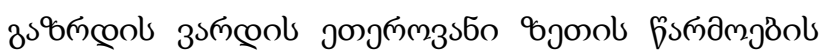

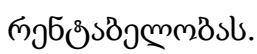

\section{domoonsceo 6sfomo}

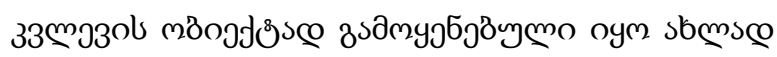

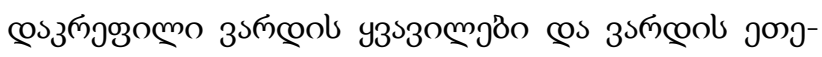

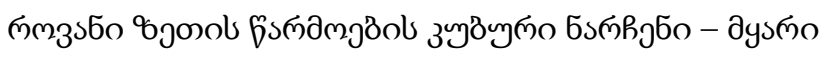
cos onbjasceo ogsos.

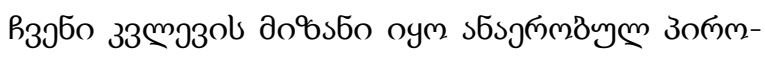

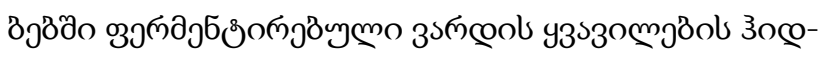

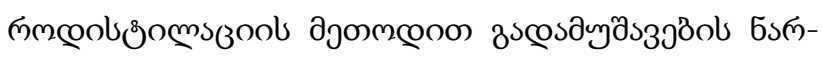

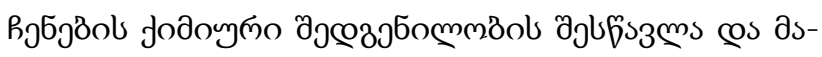

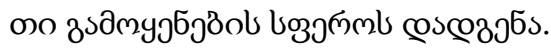

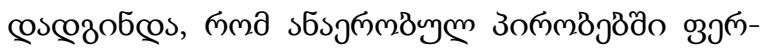

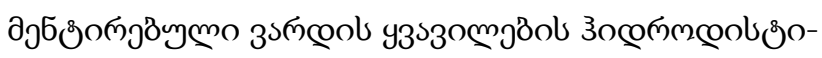

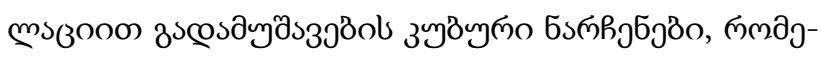

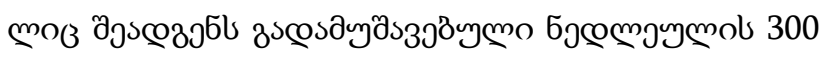

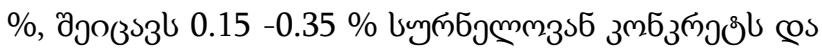

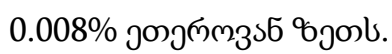


3

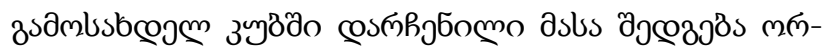

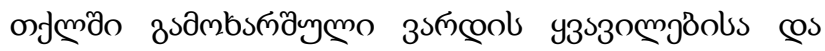

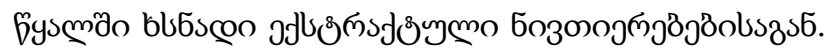

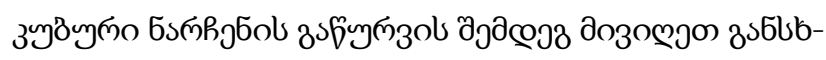

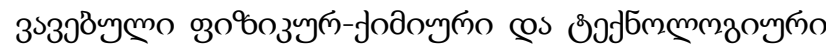

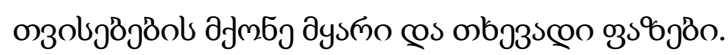

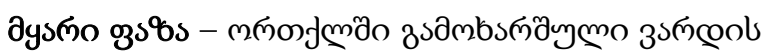

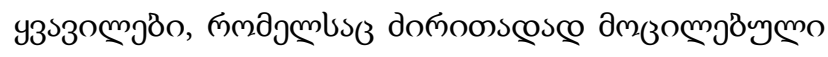

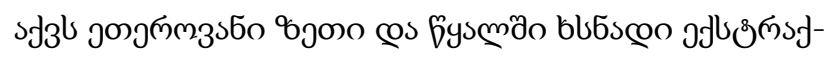

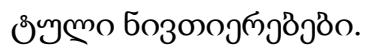

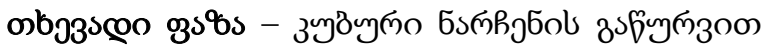

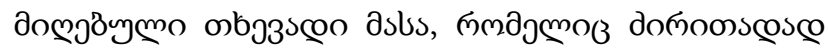

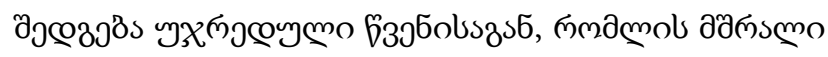

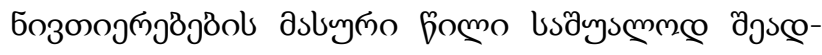
3эбl $12.5 \%$.

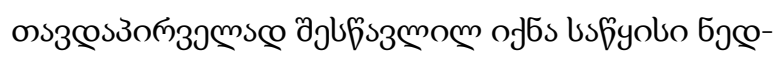

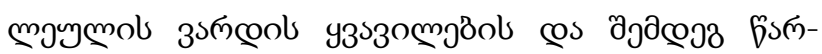

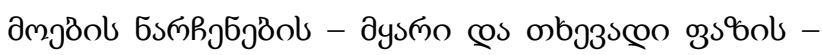

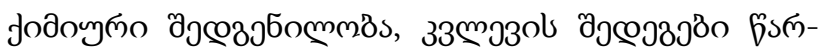

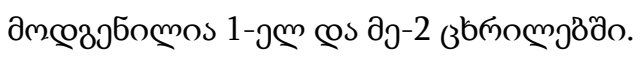

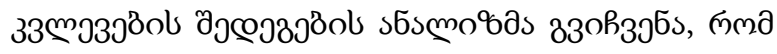

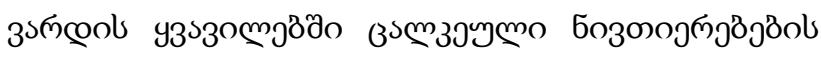

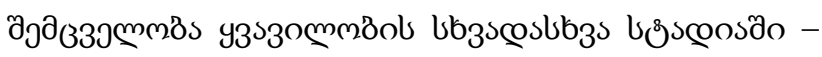

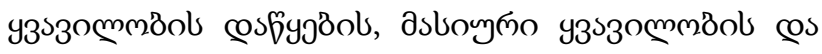

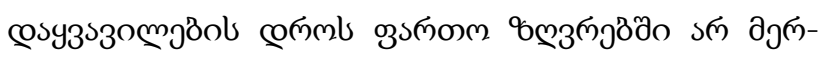

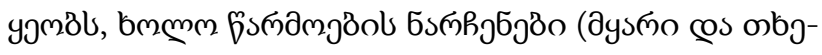

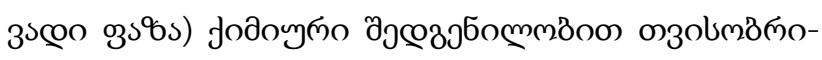

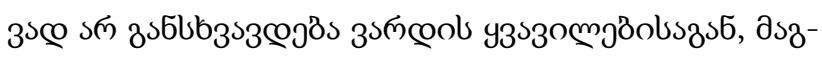

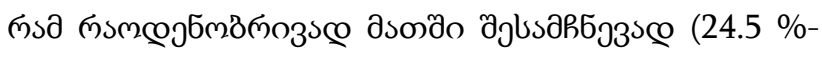
оп) дазопূ

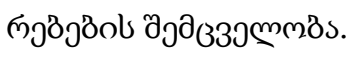

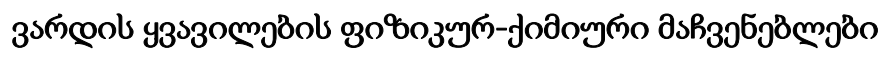

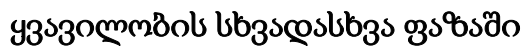

\begin{tabular}{|c|c|c|c|c|c|c|c|c|}
\hline 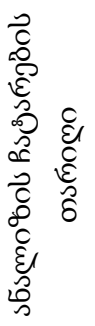 & 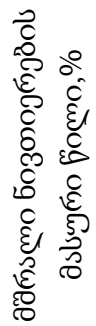 & 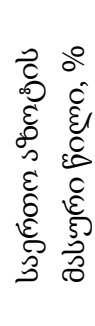 & 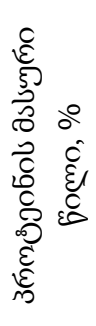 & 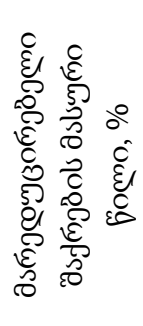 & 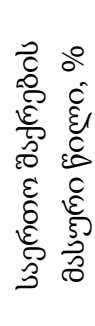 & 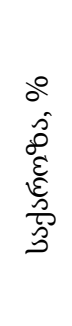 & 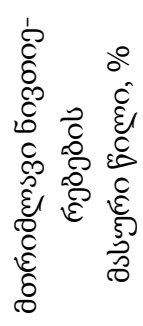 & 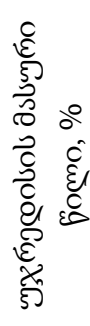 \\
\hline $24 / 5$ & 20.54 & 2.00 & 12.50 & 26.77 & 31.10 & 4.33 & 5.45 & 14.65 \\
\hline $30 / 5$ & 17.26 & 2.20 & 13.75 & 26.66 & 26.24 & 0.58 & 4.28 & 15.06 \\
\hline $6 / 6$ & 13.40 & 1.70 & 10.60 & 27.98 & 27.98 & - & 4.32 & 14.17 \\
\hline $11 / 6$ & 17.00 & 1.80 & 11.25 & 32.00 & 32.50 & 0.50 & 3.00 & 13.50 \\
\hline
\end{tabular}




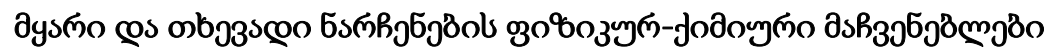

\begin{tabular}{|c|c|c|c|c|c|c|c|c|}
\hline 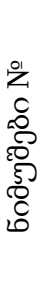 & 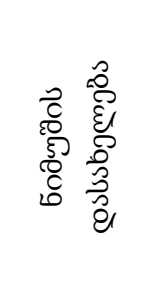 & 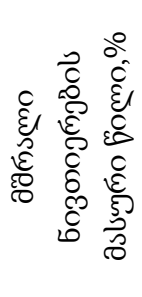 & 罢 & 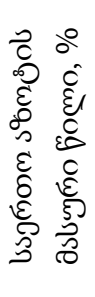 & 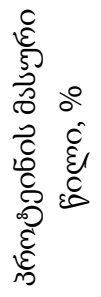 & 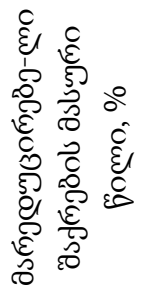 & 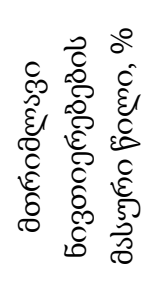 & 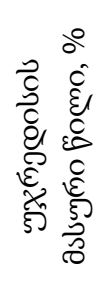 \\
\hline 1 & \multirow{4}{*}{ 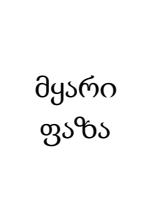 } & 20.80 & - & 1.68 & 10.50 & 3.46 & 2.40 & 18.26 \\
\hline 2 & & 18.80 & - & 1.60 & 10.00 & 1.60 & 1.60 & 19.15 \\
\hline 3 & & 27.22 & - & 1.46 & 9.2 & 4.70 & 2.20 & 12.00 \\
\hline 4 & & 23.50 & - & 1.06 & 6.62 & 6.00 & 1.57 & 10.63 \\
\hline 1 & \multirow{4}{*}{ 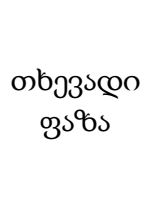 } & 11.75 & 4.5 & 0.38 & 2.37 & 5.24 & 1.19 & - \\
\hline 2 & & 8.00 & 4.3 & 0.30 & 1.87 & 3.40 & 2.60 & - \\
\hline 3 & & 17.60 & 4.4 & 0.20 & 1.81 & 2.84 & 2.00 & - \\
\hline 4 & & 12.05 & 4.4 & 0.30 & 1.87 & 3.32 & 0.15 & - \\
\hline
\end{tabular}

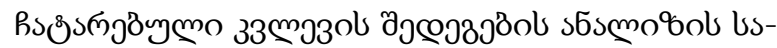

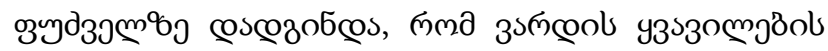

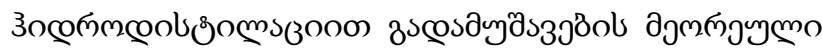

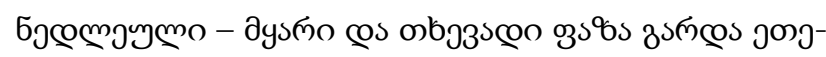

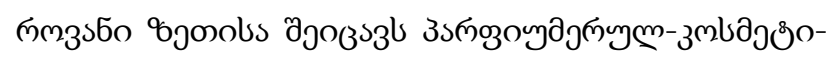

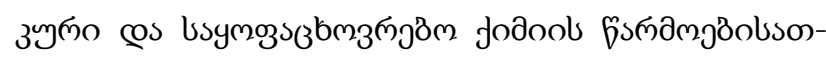

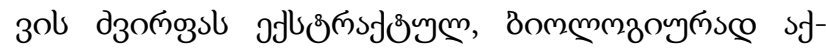
волм доподммз

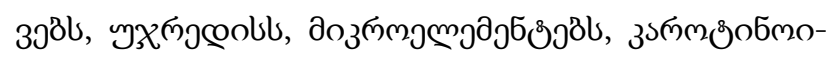
cjols cos bb3s.

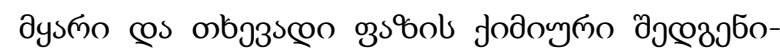

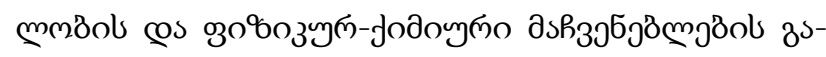

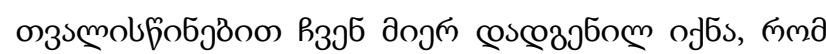

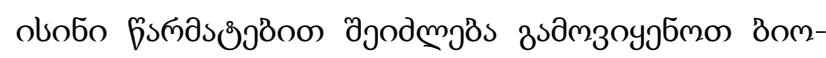

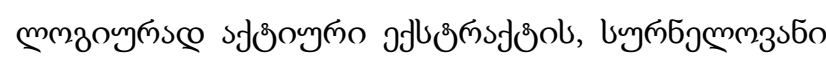

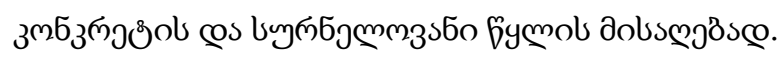

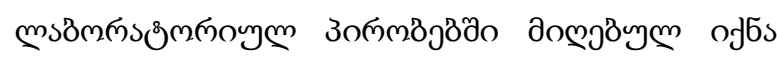

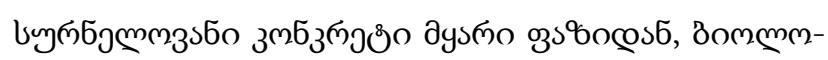

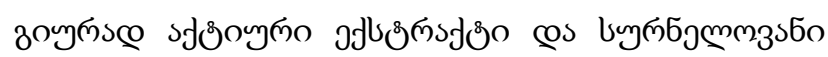

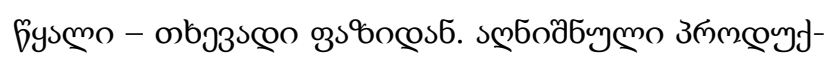

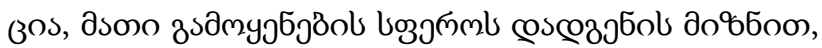

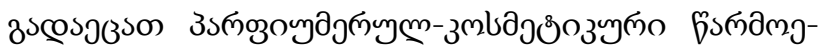

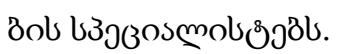

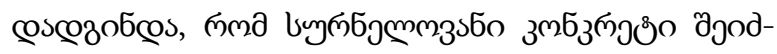

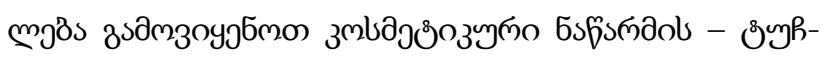

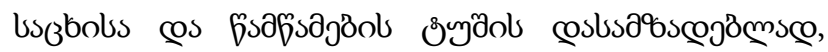

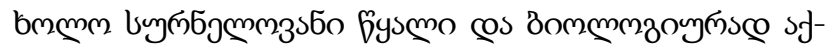

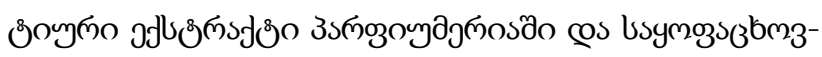

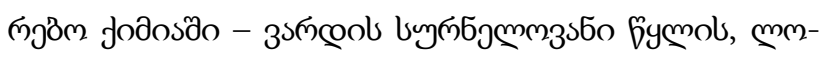

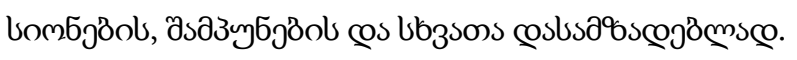

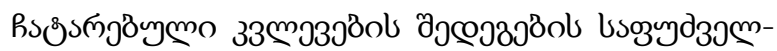

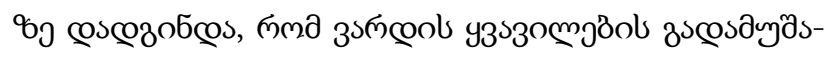

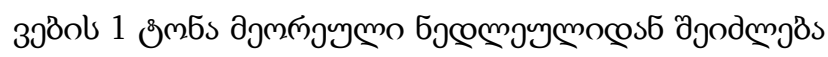

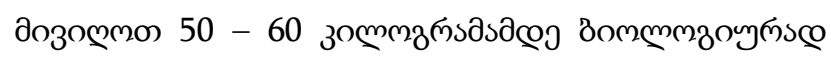

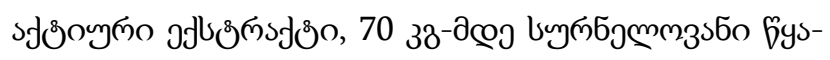

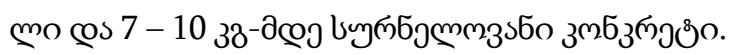

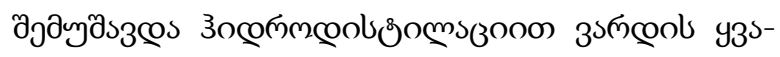

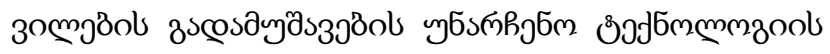

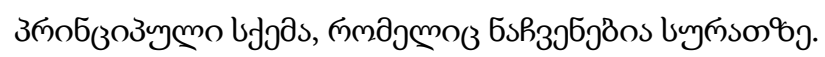




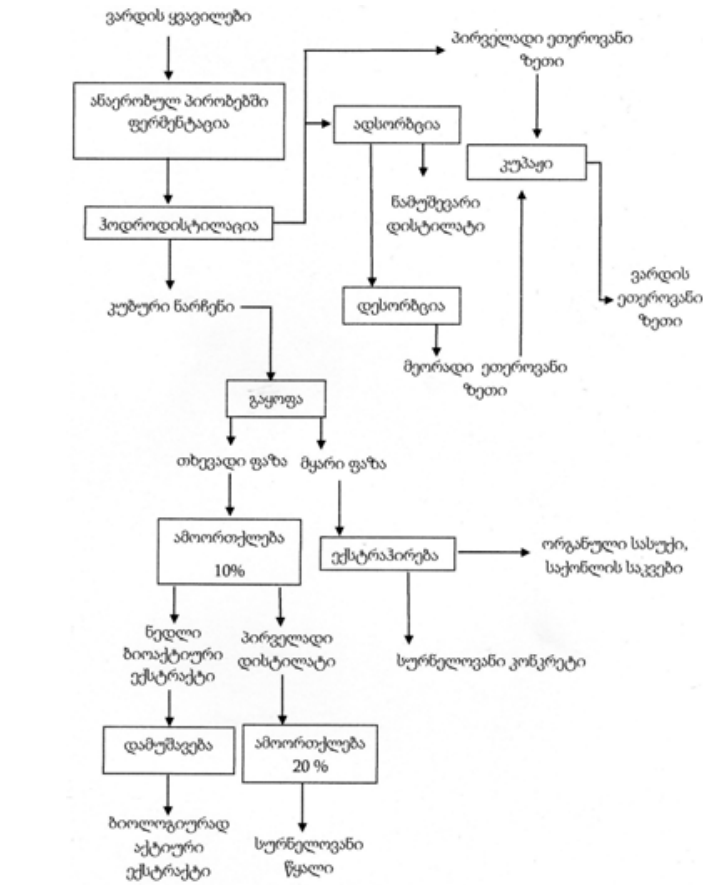

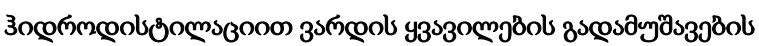

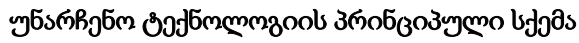

\section{coslos36s}

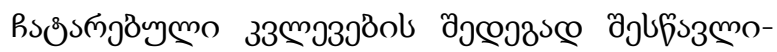

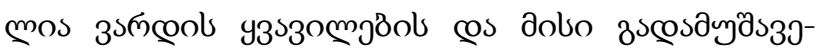

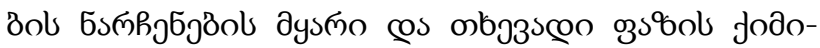

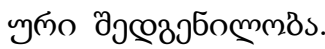

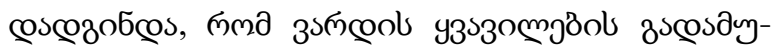

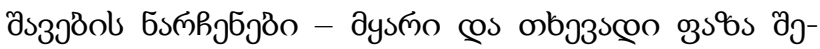

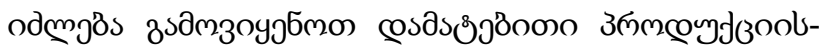

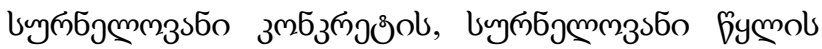

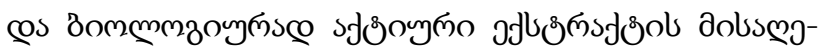

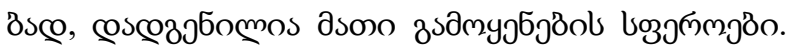

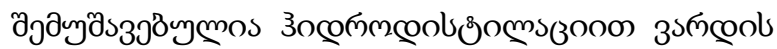

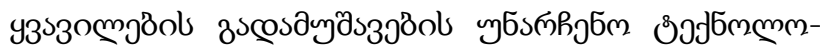

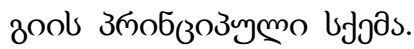

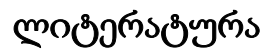

1. Baghaturia N. Essential oils of medicinal and spice-aromatic herbs. Tbilisi. 2007. (in Russian).

2. Baghaturia N. Natural wines, juices and drinks. Tbilisi. 2008. (in Russian).

3. Baghaturia N. Food products technology. Tbilisi. 2016. (in Georgian).

4. Secondary material resources of food industry. Handbook. M.: "Economics". 1984. (in Russian).

5. Zyukov D. G., Andreevich E. N., Chipiga A.P. Technology and equipment of essential oil production. M.: "Food industry”. 1979. (in Russian).

6. Iakobashvili N. Production technology of essential oils. Tbilisi. 1959. (in Georgian).

7. Sidorov I.I., Turisheva N.A. and others. Production technology of natural essential oils and synthetic aromatic substances. M.: "Legkaya i pishchevaya prom". 1984. (in Russian).

8. Chipiga A. P., Zyukov D. G., Naidenova V. P. and others. Handbook of essential-oil production technology. M.: “Legkaya i pishchevaya prom.”. 1981. (in Russian). 


\section{UDC 663.5}

SCOPUS CODE 1305

\section{Study of Rose Oil processing waste}

Nugzar Baghaturia

Luiza Kajaia

Neli Gilauri
Scientific-Research Institute of Food Industry, Georgian Technical University, 17 D. Guramishvili Ave, 0192, Tbilisi, Georgia

E-mail: nugzi@yahoo.com

Scientific-Research Institute of Food Industry, Georgian Technical University, 17 D. Guramishvili Ave, 0192, Tbilisi, Georgia

E-mail: 1.qajaia@gtu.ge

Address.Scientific-Research Institute of Food Industry, Georgian Technical University, 17 D. Guramishvili Ave, 0192, Tbilisi, Georgia

E-mail: n.gilauri@gtu.ge

\section{Reviewers:}

N. Ebelashvili, Doctor of Technical Sciences, Chief Research Scientist, Deputy Director of Scientific-Research Institute of Food Industry, GTU

E-mail: nana-ebelashvili@hotmail.com

G. Grigorashvili, Doctor of Biological Sciences, Chief Research Scientist, Scientific-Research Institute of Food Industry, GTU

E-mail: g.grigorashvili@gtu.ge

Abstract. In the article it is investigated the processing of the residues of the fermented under anaerobic conditions rose flowers by hydrodistillation.

The residues of the rose flowers consist of steamed rose flower extract, that contains water-soluble rose substances.

It's studied the chemical content of the solid and liquid phases of the processing of the rose flowers residues and the areas of their application are established as well.

It is established that the solid phase of the rose flower residues processing can be applied for the additional product aromatic concrete production and liquid phase - for the production of aromatic water and biologically active extract.

In experimental scale firstly is received aromatic concrete from the solid phase and from the liquid phase - aromatic water and biologically active extract that found application in perfumery-cosmetics production and household chemicals.

It is elaborated the principal technological scheme of waste-free processing of rose flowers by hydrodistillation.

Key words: Bioactive extract; concrete; roses; waste. 


\section{UDC 663.5}

SCOPUS CODE 1305

\section{Исследование отходов переработки эфиромасличной розы}

Нугзар Багатурия НИИ пищевой промышленности Грузинского технического университета, Грузия, 0192, Тбилиси, Проспект Д. Гурамишвили 17

E-mail: nugzi@yahoo.com

Луиза Каджая НИИ пищевой промышленности Грузинского технического университета, Грузия, 0192, Тбилиси, Проспект Д. Гурамишвили 17

E-mail: 1.qajaia@gtu.ge

Нели Гилаури НИИ пищевой промышленности Грузинского технического университета, Грузия, 0192, Тбилиси, Проспект Д. Гурамишвили 17

E-mail: n.gilauri@gtu.ge

\section{Рецензенты:}

Н. Эбелашвили, доктор наук, главный научный сотрудник, заместитель директора НИИ пищевой промышленности ГТУ

E-mail: nana-ebelashvili@hotmail.com

Г. Григорашвили, доктор биологических наук, главный научный сотрудник НИИ пищевой промышленности ГТУ

E-mail: g.grigorashvili@gtu.ge

Аннотация. Исследованы отходы переработки методом гидродистилляции ферментированных в анаэробных условиях цветков розы.

Отходы переработки цветков розы состоят из вываренного в пару сырья и экстракта, содержащего расстворимые в воде вещества.

Исследован химический состав цветов розы и отходов их переработки - твердой и жидкой фаз. Установлено, что отходы переработки цветов розы содержат дубильные и красящие вещества, белки, ароматические вещества, а также витамины, аминокислоты и др.

Установлено, что твёрдые отходы переработки цветов розы можно использовать для получения дополнительной продукции в виде душистого конкрета, а жидкую фазу для получения душистой воды и биологически активного экстракта.

Разработана принципиальная технологическая схема безотходой переработки цветков розы.

Ключевые слова: биологически активный экстрат; конкрет; отходы розы.

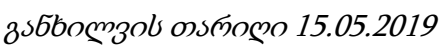

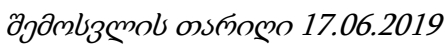

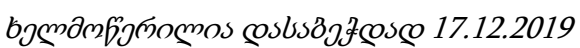

